\title{
Percepções do Processo de Diagnóstico por Estudantes de Medicina
}

\author{
Medical Students' Perceptions of the \\ Diagnostic Process
}

Dejano T. Sobral ${ }^{1}$

WEURS-CHAVE:

Dhansice;

Hethls ates de Medicna;

Fharrcào Médica;

analuçà

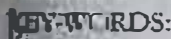

Pingmores:

Jordmbirialitel:

Eduaton, Miticit

Frimation.

sorsicito em: $08 / 10 / 2003$

Apunialis em: 11/03/2004

\begin{abstract}
RESUMO
O propósito deste estudo foi analisar o espectro de respostas dos alunos de medicina da Universidade de Braslia ao Inventário de Racioclnio Diagnóstico (IRD). O inventáro foi aplicado na fase de inicziasâa clinica a 677 alunos de ambos os sexos, no decorjer de 11 anos. Foram utilizados procedimentos estatsticos para identificar conjuntos de itens interrelacionados e grupos com respostas similares, bem como diferenças entre grupos e suas relaçoes com atributos distintos. Os resultados das análises revelaram seis dimensdes relevankes do IRD, denominadas: procedimento diligente, apreensäo fácil,

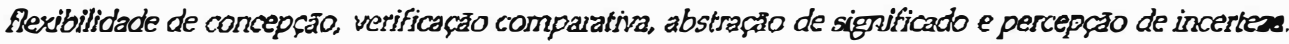
Seis grupos de classificapáo foram identificados pelo espectio de variaço das dimensoes. Os valores das indices do IRD $e$ as respectivas afiliaģos dos alunos aos grupos se relacionaram com a epoca de inserça no estudo, o gradiente de rendimento cognitivo na iniciação dinica, o nivel de eficácia percebida e a frequência de atividade de monitoria nos dois semestres seguintes à aplicacao do IRD. Em conclusāo, os estudantes diferem nas percepços, do processo diagnóstico, inclusas no IRD e as

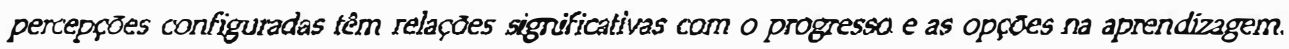

\begin{abstract}
The purpose af this study was to assess the patterns of medical students' responses to the Diagnostic Thinking Inventory (DTD at the University of Brasilia. The inventory was adninistered within an IIyear timeframe to 677 students of both sexes early in their clinical experience. Statistical procedures were used to identify interrelated item sets and groups with similar response patterns, as well as differences between groups and their relationships with distinctive features. Results of the analyses revealed six salient DTT dimensions designated as follows: diligent procedure, easy grasp, flevibility of conception comparative appraisal, abstraction of meaning, and perception of uncertainty. Six student classification groups were identified based on clustering of the dimension indices. Studenw' DTI indices and respective group affiliation were related to their indusion period in the study timeframe, early clinical achievement-gradient, self-efficacy level, and iwo-semester frequency of peer tutoring after the DTI appraisal. In conchusion, medical students differ in their perceptions of the diagnostic process as entailed by the DTh, and the patterns of perceptions have meaningful relationships with their leaming progress and options.
\end{abstract}




\section{INTR்ODUÇÃO}

O diagnóstico dínico é um elemento distintivo da expertise em diferentes campos da prática médica e seu processo de diferenciação tem importância para o estudo da formaçào profissional. Evidências de pesquisa indicaram que aprendizes e profissionais utilizam similarmente vários elementos do processo diagnóstico, mas com conteúdo qualitativamente distinto e freqüência variável ${ }^{1}$. A caracterização e a medida desses elementos do processo diagnóstico tem gerado interesse, considerando a pertinência para intervençōes educatjvas em diferentes opçōes de delineamento curricular.

Bordage, Grant e Marsden construíram o Inventário de Raciocínio Diagnóstico (IRD) visando à avaliação e ao aprimoramentopotencial do raciocínio diagnóstico ${ }^{2}$. O instrumento está fundamentado nas pesquisas conduzidas por Borda$\mathrm{ge}^{3.4}$, Gale e Marsden ${ }^{5}$ e Grant e Marsden ${ }^{\mathrm{j} .6}$, e abrange facetas críticas do processo diagnóstico, entre as quais: aspectos salientes, reinterpretaçăo de achados, modo de inquirir, mudança de percep̧̧ão, julgamento reversível, relações abstratas e acesso à estrutura do conhecimento na memória. Seus proponentes postularam que o IRD media duas categorias de processo: grau de flexibilidade no raciocinio e grau de estrutura do conhecimento na memória. O estudo original revelou a consistência interna do instrumento e os aspectos de sua sensibilidade discriminante; observaram-se, em particular, diferenças expressivas nos valores do IRD entre grupos representativos de etapas progressivas da formação médica. Trabalhos oriundos de contextos institucionais diversos confirmaram a validade de conteúdo, mostraram a estabjlidade temporal e indicaram a utilidade potencial do instrumento no exame do processo diagnóstico em diferentes circunstâncias ${ }^{7-10}$.

Estudos realizados com estudantes de medicina apontaram correlações positivas entre os escores do IRD e medidas objetivas de rendimento cognitivo, bem como entre tais escores e indicadores subjetivos de construção/processamento da aprendizagem. Em particular, foram observadas associaçōes releyantes entre os escores do IRD e o grau de autoconfiança como aprendiz, a extensão de valorização do aprendizado e 0 nível de autodeterminação percebida na aprendizagem ${ }^{11}$.

Outros resultados de interesse revelaram que o incremento dos escores do IRD, observado no progresso da iniciação clínica, relacionava-se diretamente no nível de autodeterminação percebida por aprendizes no raciocínio diagnóstico. Esses e outros achados indicam que o IRD reflete a noção pessoal de capacidade no processo diagnóstico, que envolve conhecimento e habilidades espećíficas, mas depende de diferentes fatores no contexto do aprendizado e de atitudes do aprendiz ${ }^{12}$. Essa relação foi interpretada em termos do principio da auto-eficácia. Segundo Bandura, a percepção da auto-eficácia refere-se às crenças pessoais na capacidade de organizar e efetuar os atos requeridos para conseguir urna realizaçăo e tem caráter preditivo em relação ao funcionamento psicossocial do indivíduo ${ }^{13}$. No processo diagnóstico, a expectativa de auto-eficácia deriva das convicçōes pessoais do aprendiz quanto a sua competência em organizar e efetuar as condutas requeridas para a consecução do diagnóstico clénico. Sảo fontes de informação para a percepção da auto-eficácia as experiências diretas ou vicariantes, a persuasảo verbal e os estados afetivo e fisiológico do aprendiz. Esses elementos sảo inerentes ao contexto de uso do processo diagnóstico nas condiçōes de aprendizagem durante a iniciação clínica da formação médica.

Os achados de diversos estudos anteriores sugerem a possibilidade de que os aprendizes difiram tanto na intensidade de expressão quanto na configuração dos elementos do processo de diagnóstico clínico embutidos no IRD. Como se caracteriza e em que importa esse espectro de configuraçỏes? Esta questão foi examinada no presente estudo, que faz parte de linha de investigação sobre o desenvolvimento do processo diagnóstico, realizado na situação real da aprendizagem clínica. Partiu-se da hipótese de que fatores relacionados ds circunstâncias do aprendizado na fase de iniciação clínica influenciam o espectro de respostas ao IRD e suas relaçð̉es com desfechos acadêmicos. Nessa perspectiva de análise, aproveitou-se a base de dados da experiência de aplicaçăo do instrumento de Bordage e colaboradores em turmas consecutivas de estudantes de medicina na Universidade de Brasília. Tre̊ objetivos dessa análise são realçados no relato.

1) Identificar as dimensōes do processo diagnóstico que estảo implícitas nas respostas do conjunto dos estudantes ao Inventário de Racioćnio Diagnóstico (IRD).

2) Averiguar as diferenças nas dimensðes do processo entre grupos de estudantes classificados pelo espectro de respostas ao IRD.

3) Examinar as relaçōes entre os grupos de classificação do IRD e quatro indicadores e atributos pertinentes aos estudantes: a época de inserção no estudo, o nível de eficácia pessoal no processo, o gradiente de rendimento cognitivo na iniciação clínica e a realizaçảo subseqüente de atividade de monitoria em disciplina.

\section{METODOLOGIA}

\section{Delineamento}

Trata-se do estudo retrospectivo, constituído de dados de coortes consecutivas, com elementos de acompanhamento no periodo de um ano. 


\section{Sujeitos}

A população-alvo do estudo compreendeu estudantes do curso de medicina da Universidade de Brasília, no peráodo de $1 / 92$ a 2/02. O critério de inclusão foi o registro dos estudantes no sexto semestre do curso, por amostragem consecutiva de 22 turmas de ingresso semestral ${ }^{14}$. Do total de 691 alunos $(56,7 \%$ masculinos) inclứdos pelo critério, 14 (52,3\% masculinos) não completaram o inventário e foram excluîdos das análises. No ano de acesso ao sexto semestre, a média da idade dos 677 alunos credenciados foi 22,09.

\section{Contexto}

A iniciação clínica formal no curso de medicina da Universidade de Brasília começa no qquinto semestre, quando os estudantes cursam a disciplina de semiologia e desenvolvem $o$ aprendizado da entrevista e do exame físico lidando diretamente com pacientes. No sexto semestre, os estudantes realizam exames clínicos completos e são orientados a usar e desenvolver o raciocínio clínico nas enfermarias de clínica médica e clínica cirúrgica do Hospital Universitário de Brasília. O conteúdo da experiência clínica dos estudantes se refere, portanto, a uma mulkiplicidade de problemas clínicos de yariável complexidade, em adultos.

\section{Medidas e Procedimentos}

A versão em Português do Inventário de Raciocínio Diagnóstico (IRD), utilizada neste estudo, consta de anexo do trabalho de Sobral ${ }^{12}$. O IRD tem 41 itens. Cada item do inventáxio consiste em um enưnciado seguido por duas afirmações opostas separadas por uma escala de seis pontos do tipo diferencial semântica, conforme o exemplo a seguir.

(Item 33) Quando me vem uma idéia geral sobre o problema do paciente:

Posso usualmente passar para um diagnóstico específico Acho difícil colocá-la em em termos específicos

O respondente deve assinalar o ponto na escala que melhor expressa sua posição entre as afimações opostas. Cada itern tem uma pontuação máxima de 6 , na dependência da proximidade do ponto com a afirmação mais claramente associada ao padrão definido por peritos ${ }^{2}$. O coeficiente de consistência interna do IRD (alfa de Cronbach) foi 0,83 . No presente estudo, os escores do inventário foram expressos na forma de indices médios (quociente da soma da pontuaçăo dos itens, na escala de 1 a 6 , dividida pelo número de itens), Assim, o indice médio global corresponde à soma das pontuações dos 41 itens, dividida por 41 e tem limites mínimo e máximo, portanto, na faixa de 1,0 a 6,0. Na aplicação do inventário adicionou-se uma queståo expressa em escala global de quatro pontos para aferir a percep̧̧ão de autodeterminação no raciocínio diagnóstico. A extensão de autodeterminação, nessa escala, se estendia de restrita (necessitando amadurecimento, incentivo, treinamento e feedback para desenvolvimento de raciocínio clínico eficaz), até máxima (capaz de raciocínio clínico eficaz mesmo em condições adversas de tempo e contexto de desempenho).

$O \mathbb{R D}$ foi aplicado entre as $10^{\mathrm{a}}$ e $12^{\mathrm{a}}$ semanas do período letivo, com referência ao modo geral de raciocínio diagnóstico, levando em conta a natureza da experiência clínica dos aprendizes. Sua aplicação, no seguimento de várias discussões de casos clínicos, serviu como exercício educativo e cada aprendiz teve acesso a um retomo comentado de suas respostas, realçando os indicadores de flexibilidade de raciocinio e estrutura do conhecimento na memória.

$O$ indice de rendimento acadêmico dos estudantes foi calculado para o quinto e sexto periodos do curso, com base nas mençōes e no número de créditos das respectivas disciplinas obrigatórias. O gradiente de rendimento cognitivo foi obtido pela comparação emparelhada desses índices consecutivos.

Apurou-se, em acréscimo, o registro de atividade de monitoria no ano seguinte ao exercício do IRD (sétimo e oitavo períodos do curso). Para a grande maioria dos estudantes, a realização de monitoria, nessa fase do curso, tem por objeto disciplinas cursadas no ano anterior - entre as quais semiologia, clínica médica e clínica cirúrgica.

Dados do questionário de orientação (incluindo estilo de aprendizagem e grau de autoconfiança como aprendiz), completado ao término do primeiro ano do curso, estavam disponiveis para 667 alunos ${ }^{11}$. Essas informaçōes não foram obtidas no caso de outros 10 alunos transferidos de outras instituiçōes ao término da fase pré-clínica do curso.

\section{Análise}

Quatro procedimentos estatisticos principais foram utilizados: (a) análise fatorial para identificar variáveis subjacentes (fatores) que explicam as correlaçōes entre os itens do IRD; (b) análise de agregação hieránquica (hierarchical cluster analysis), para obter uma classificação de casos similares, segundo as variáveis identificadas; (c) testes $t$, ou análises de variância, para aferir diferenças entre médias de grupos; (d) análise de regressão logística para verificar a associação entre um fator explanatório e a variável binária de desfecho, quando outros fatores são controlados. Os textos de Norman e Streiner ${ }^{15}$ e de Glantz e Slinket ${ }^{16}$ orientaram os planos e procedimentos de análise. A análise foi realizada no programa SPSS 10.0.

\section{RESULTADOS}

A análise fatorial (método de componentes principais, com rotação equamax) das respostas aos 41 itens do IRD, no con- 


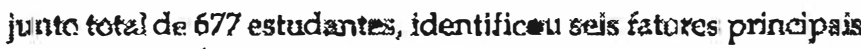

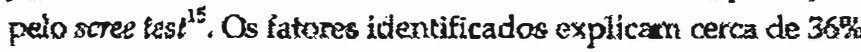
da variankia total das respostas aos iters do IRD e correspon-

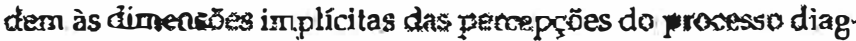
nő́tico que estăo embutidias nas respostas ao inventário.

As seis dimensơ fatoriais foran assim derominadas (na ordem decrescente de variància explicada):

(1) Procedimento tiligente, 10 itens; fndice média: 3,20 ;

(2) Apreensầo fácil, 9 itens; indice médio: 4,48;

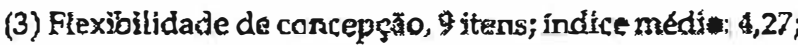

(4) Verificaçăo comparativa, 4 itens; indice médio: 4,63;

(5) Abstração de signiobado, 4 itenși indice médio: 4,16;

(6) Percepção de incerteza, 5 itens; Iriclice méd:́: 4,31.

$O$ s indices madios diferem significantemente sntre si. exceto na comyzraçẵo entre a terceira e spxta dímensóes, spa nas na dimensăo de evaliaçă comparativa houve diferença significaste entre sixos, sendo o indice maior para o feminino $\{t=2,3 ; d f=675 ; p=0,024\}$. Todas as dimenszes, exceto

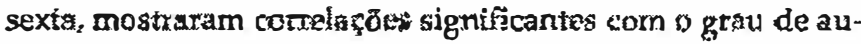

toconfiança como ap̧rendiz; a dimensăo de procedlumento di ligente mastrou a correlaça mais forte $(r=0,31 ; 0<0,000)$. A Tabela 1 apresenta itens representativon de cada diniensäo fatorial

Poram coservadas dúferenças signifficanles entre os indi ces médies das dimensŏes do [थD para os estudantes agrupa dos segurido dias époras histzricas de inserção no sexto pe riodo do curyo: sernestre $1 / 32$ a $1 / 97$ ( $n=292 / 54,6$ \% mascus linos). ou semestre 2,197 a $2 / 02(n=385$ / $57,4 \%$ masculinus Estudantes inseridos na epoca mais recente apresentaram valors significantemente maiore do indice métio global a dos indices medios dos conjuntos de itens carrespondentes âs dimensžc de procedimento diligente, abstração de signi-

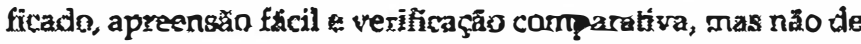

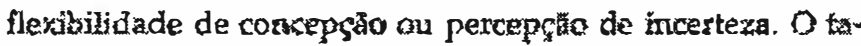
rnantio do efeito da época historica fui pequeno (effect sizem 0,40) para o údize mélio global.

As variăyeis das dimensôtes fatortais foram utilizadas para

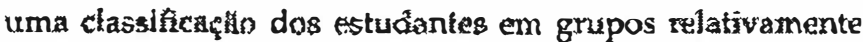

Tabela 1

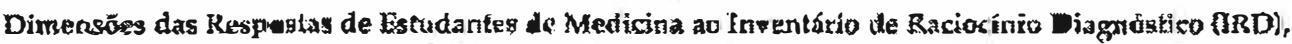
Obtidas por Ansilise Fatorial $(\mathbf{N}=677)^{*}$

\begin{tabular}{|c|c|c|}
\hline Dimersges do IRD & Média (appl & HeHs representutiras no iRD \\
\hline $\begin{array}{l}\text { 1.Procediunento cilligente } \\
\text { (1D itens) }\end{array}$ & $3,90(0,30)$ & 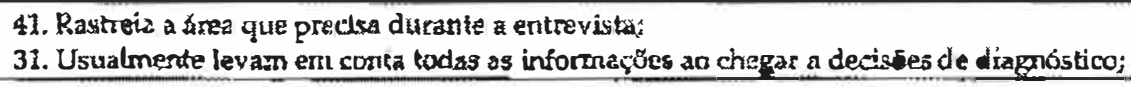 \\
\hline $\begin{array}{l}\text { 2. Apreensāo tácil } \\
\text { (9 iturns) }\end{array}$ & $4,48\{0,55\}$ & $\begin{array}{l}\text { 18. Antecipa mentalmen te passiveiz sintomas e sinais; } \\
\text { 11. Itens-chave de informaçăo parecem saltar aos othos; }\end{array}$ \\
\hline $\begin{array}{l}\text { 3. Dlexibilidade de concepcä̀ } \\
\text { (9 iters) }\end{array}$ & $4,27(0,65)$ & 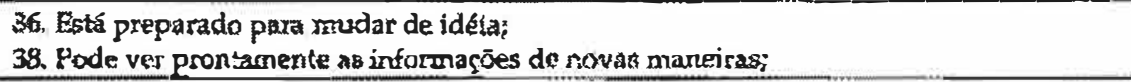 \\
\hline $\begin{array}{l}\text { 4. Verifica yölo comparativa } \\
\text { (4 itens) }\end{array}$ & $4,63(0,76)$ & $\begin{array}{l}\text { 40. Compara e contrasta possjveis diagnósticos; } \\
\text { 30. Avmlia periodicamente os dadon e as próprias ideias; }\end{array}$ \\
\hline $\begin{array}{l}\text { 5. Aibstraçäo de sigrificedo } \\
\text { (4 iters) }\end{array}$ & $4,0,6(0,52)$ & 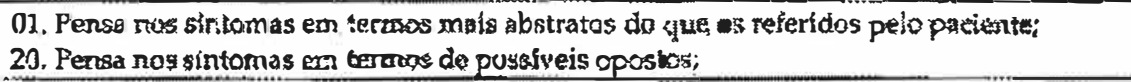 \\
\hline $\begin{array}{l}\text { 6. Fercepgâo de incerteza } \\
\text { (.5 iteng) }\end{array}$ & $4,31(9,65)$ & 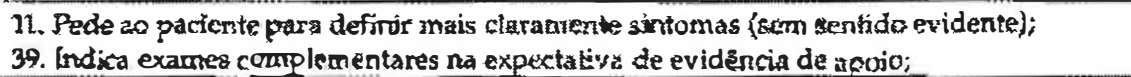 \\
\hline
\end{tabular}

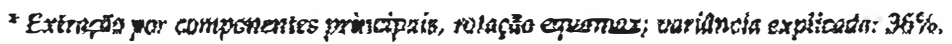

Tabela 2

Tegtes de Diferengas entre Índices Mt́dios das Dimensōes do Inventário da Raciocinio Diagnéstico (IRßD) em Estudanfes de Medicina Agrupados Segundo Daso Eperas da Irserça no Sexto Semestre do Curgo

\begin{tabular}{|c|c|c|c|c|}
\hline \multirow{2}{*}{ Wimensoes do ling } & \multirow{2}{*}{ 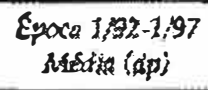 } & \multirow{2}{*}{$\begin{array}{l}\text { Expeco 2,97-2/02 } \\
\text { iredia (dp) }\end{array}$} & \multicolumn{2}{|c|}{ Tèstes : } \\
\hline & & & Diferença & $p$ \\
\hline 1. Procedimento diligente & $3.70\{0,68\}$ & $4,05\{0,67\}$ & 1,36 & 0,000 \\
\hline 2. Aprtensão făcil & $4,35\langle 0,33\}$ & $4,58(0,54)$ & 0.23 & 0,000 \\
\hline 3. Flexibilidade de concepşa & $4,30(0,67)$ & $4,25(0,63)$ & $-0,05$ & $-0,274$ \\
\hline 4. Verificaço womparativa & $4,57(0,83)$ & $4,69\{0,74\}$ & 0,12 & 0,047 \\
\hline 5. Aligtracta de significado & $389(0,50)$ & $4,39\{0,81\}$ & 0,31 & 0,000 \\
\hline 6. Yeccespio de incertezá & $4,30(0,65)$ & $+32(0,65)$ & 0,02 & 0,582 \\
\hline IRD total & $4,15(0,11)$ & $1,32(0,42)$ & 0,17 & 0,000 \\
\hline
\end{tabular}


Tabela 3

Testes de Diferenças entre frndices Médios das Dimensōes da IRD em Estudantes de Medicina Agrupados Segundo Classificação por Anślise de Agregação Hierárquica

\begin{tabular}{ccccccc}
\hline $\begin{array}{c}\text { Dimensđes do IRD } \\
(a)\end{array}$ & $\begin{array}{c}A(n=96) \\
\text { Média }(d p)\end{array}$ & $\begin{array}{c}B(n=271) \\
\text { Média }(d p)\end{array}$ & $\begin{array}{c}C(n=62) \\
\text { Média (dp) }\end{array}$ & $\begin{array}{c}D(n=77) \\
\text { Média (dp) }\end{array}$ & $\begin{array}{c}E(n=109) \\
\text { Média (dp) }\end{array}$ & $\begin{array}{c}F(n=62) \\
\text { Média }(d p)\end{array}$ \\
\hline $\mathbf{1}$ & $4,85(0,30)$ & $4,10(0,45)$ & $4,22(0,31)$ & $3,40(0,33)$ & $3,23(0,50)$ & $3,05(0,45)$ \\
\hline 2 & $4,99(0,39)$ & $4,58(0,41)$ & $4,76(0,45)$ & $4,36(0,44)$ & $4,24(0,35)$ & $3,55(0,40)$ \\
\hline 3 & $4,91(0,45)$ & $4,56(0,40)$ & $3,61(0,34)$ & $4,38(0,41)$ & $3,51(0,50)$ & $3,92(0,49)$ \\
\hline 4 & $5,25(0,55)$ & $4,81(0,69)$ & $4,33(0,71)$ & $4,48(0,75)$ & $4,16(0,62)$ & $4,24(0,71)$ \\
\hline 5 & $4,51(0,89)$ & $4,10(0,82)$ & $4,25(0,54)$ & $3,57(0,73)$ & $4,04(0,74)$ & $3,57(0,59)$ \\
\hline 6 & $4,56(0,66)$ & $4,32(0,70)$ & $4,46(0,56)$ & $4,08(0,59)$ & $4,16(0,60)$ & $4,27(0,47)$ \\
\hline IRD total & $4,86(0,23)$ & $4,40(0,21)$ & $4,25(0,18)$ & $4,03(0,17)$ & $3,80(0,30)$ & $3,67(0,24)$ \\
\hline
\end{tabular}

(a) Identificaçäo na Tabela 2.

Testes Kruskal-Wallis: $p<0,000$ na comparaçăo de grupos em cada dimensāo.

homogêneos, mediante análise de agregação hierárquica (hiezarchical cluster analysis). Obteve-se uma solução estável de seis grupos com distribuição sexual equivalente e tamanho desigual: A (96), B (271), C (62), D (77), E (109) e F (61). A Tabela 3 identifica tais grupos, que diferem significativamente entre $s i$ pelo valor decrescente (na ordem alfabética) do índice médio global e, também, pelos valores relativos dos índices médios das dimensões do IRD. Os grupos $\mathrm{A}$ e $\mathrm{C}$ têm indices médios mais elevados que os demais grupos nas dimensões de procedimento diligente, apreensāo fácil, abstração de significado e percepção de incerteza. Em acréscimo, os graus de autoconfiança como aprendiz dos grupos $\mathbf{A}, \mathbf{C}$ e $\mathbf{B}$ foram significativamente superiores aos graus dos grupos $\mathbf{E}, \mathrm{F}$ e $\mathrm{D}$.

Observou-se uma associação significante entre as proporçōes de estudantes nos grupos de classificação do IRD e a época histórica de inserção no sexto periodo do curso. Essa relação é atribuivel, principalmente, ao aumento das proporçס̃es de estudantes dos grupos $\mathbf{A}$ e $\mathbf{C}$ na época mais recente de inserção.

Tabela 4

Distribuição de Estudantes de Medicina por Grupos de Classificação do IRD em Duas Epocas Históricas do Curso

\begin{tabular}{ccc}
\hline $\begin{array}{c}\text { Grupos de } \\
\text { classificaçăo }\end{array}$ & $\begin{array}{c}\text { Epoca 1/92-1/97 } \\
n(\%)\end{array}$ & $\begin{array}{c}\text { Epoca } 2 / 97-2 / 02 \\
n(\%)\end{array}$ \\
\hline $\mathbf{A}(\mathrm{n}=96)$ & $26(27,1)$ & $70(72,9)$ \\
\hline $\mathbf{B}(n=271)$ & $117(43,2)$ & $154(56,8)$ \\
\hline $\mathbf{C}(n=62)$ & $16(25,8)$ & $46(74,2)$ \\
\hline $\mathbf{D}(n=\pi 7)$ & $45(58,4)$ & $32(41,6)$ \\
\hline $\mathbf{E}(n=109)$ & $51(46,8)$ & $58(53,2)$ \\
\hline $\mathbf{F}(n=62)$ & $37(59,7)$ & $25(40,3)$ \\
\hline Total $(n=677)$ & $292(43,1)$ & $385(56,9)$ \\
\hline
\end{tabular}

Qui-quadrado $=32,5 d f=5 p<0,000$
Verificou-se que os indices globais do IRD eram significativamente distintos entre os estudantes na dependência do nivel de autodeterminação (ou eficácia pessoal) percebida no processo de raciocínio diagnóstico. Os seis itens relacionados a seguir foram identificados, em análise de regressão logística, como preditivos significantes da distinção entre o nível Iimitado (restrito ou parcial), ou nível extenso (amplo ou máximo) de autodeterminação percebida no processo diagnóstico.

(Item 20) Ao considerar os sinais e sintomas do paciente (penso neles em termos de possiveis opostos - e.g., progressivo $X$. súbito; espástico $X$ flácido; unilateral $X$. bilateral).

(Item 12) Ao considerar possibilidades de diagnóstico em casos clínicos (habitualmente estou na pista certa).

(Item 18) Quando um possivel diagnóstico surge na minha menke durante uma entrevista (usualmente vejo-me antecipando mentalmente possiveis sintomas e sinais que combinam com tal hipótese).

(Item 27) Em relação a um diagnóstico qque eu tenha finalmente feito (usualmente eu tenho poucas dúvidas).

(Item 23) Quando estou tirando uma história, acho que (posso obter novas idéias por simples repasse dos dados existentes na minha mente).

(Item 41) Em termos do modo como eu conduzo uma entrevista (usualmente eu rastreio a área que preciso durante a entrevista\}.

O índice médio de pontuação calculado para os seis itens - denominado doravante eficácia diagnóstica presumida diferiu significantemente entre os seis grupos de classificação hierárquica do IRD (anova one-way, $F=127,1 p=0,000$ ). A Tabela 5 mostra os valores desses índices de eficácia presumida para cada grupo e a relação respectiva com a proporção de 
Tabela 5

Diferenças no Índice de Eficácia Diagnóstica Presumida e na Percepção de Autodelerminação no Processo Diagnóstico entre Estudantes de Medicina em Grupos de Classificação do IRD

\begin{tabular}{lcc}
\hline Grupos de classificafâo & $\begin{array}{c}\text { Eficácia diagnóstica (a) } \\
\text { Média (dp) }\end{array}$ & $\begin{array}{c}\text { Autodeterminaçao (b) } \\
\text { Nivel extenso (\%) }\end{array}$ \\
\hline$A(n=96)$ & $4,97(0,42)$ & 62,9 \\
\hline$B(n=271)$ & $4,45(0,42)$ & 31,2 \\
\hline$C(n=62)$ & $4,48(0,45)$ & 30,4 \\
\hline$D(n=77)$ & $3,96(0,41)$ & 15,6 \\
\hline$E(n=109)$ & $3,81(0,48)$ & 13,8 \\
\hline$F(n=62)$ & $3,53(0,47)$ & 4,0 \\
\hline
\end{tabular}

(a) Calculado pela pontuaçăo médía dos itens 12, 18, 20, 23, 27 e 41 do IRD. (b) Proporf̧̄o de estudan tes com nivel extenso de autodeterminaçäo percebida no processo dingnóstico.

estudantes referindo nivel extenso de autodeterminação no processo diagnóstico. Quatro conjuntos foram diferenciados (A, B+C, D+E, F) por testes de comparação múltipla.

Observaram-se, também, diferenças expressivas na comparaçāo emparelhada dos índices de rendimento acadêmico nos quinto e sexto semestres do curso, entre os estudantes agrupados segundo a classificação de agregaçăo hierárquica do IRD. Houve crescimento significante no rendimento médio dos estudantes de quatro dos seis grupos. Os dois grupos sem crescimento significante (E e F) sāo aqueles que apresentam valores menores tanto no índice global quanto, especialmente, em duas dimensões do IRD: procedimento diligente e apreensāo fácil. A Tabela 6 revela os dados de comparaçāo.

A Tabela 7 mostra as diferenças nas proporçōes de estudantes que realizaram atividade de monitoria em disciplinas no sétimo e/ou oitavo semestre do curso. Observou-se uma associação significante entre a categoria de classificação do
IRD e a freqüuencia da atividade de monitoria. Quatro grupos (A-D) mostraram proporções crescentes de casos na faixa de freqǚência de monitoria de 0 a 2. Para um grupo (F), as respectivas proporçães foram decrescentes: a parcela maior de alunos nāo realizou atividade de monitoria e a menor parcela realizou duas atividades consecutivas.

Tabela 7

Distribuição de Atividade de Monitoria Realizada por Estudantes de Medicina no Sétimo e Oitavo Períodos do Curso, Segundo Grupos de Classificação do IRD

\begin{tabular}{lccc}
\hline \multirow{2}{*}{ Grupos de classificą̧ao } & \multicolumn{3}{c}{ Frequiència de monitoria } \\
\cline { 2 - 4 } & 0 & 1 & 2 \\
\hline$A(n=96)$ & $21(21,9)$ & $37(38,5)$ & $38(39,6)$ \\
\hline$B(n=269)$ & $70(26,0)$ & $96(35,7)$ & $103(38,3)$ \\
\hline$C(n=62)$ & $16(25,8)$ & $21(33,9)$ & $25(40,3)$ \\
\hline$D(n=77)$ & $18(23,4)$ & $25(32,5)$ & $34(44,2)$ \\
\hline$E(n=108)$ & $30(27,8)$ & $53(49,1)$ & $25(23,1)$ \\
\hline$F(n=61)$ & $27(44,3)$ & $22(36,1)$ & $12(19,7)$ \\
\hline Total $(N=673)$ & $182(27,0)$ & $254(37,7)$ & $237(35,2)$ \\
\hline
\end{tabular}

Seis estudantes não tinham registro no sétimo e oitavo perfodos. Qui-quadrado $=25,1$ df $=10 p=0,005$.

\section{DISCUSSÃO}

Uma vantagem do uso do IRD nos estudos sobre diagnóstico clínico em aprendizes é a facilidade de aplicaçāo. $O$ instrumento pode ser respondido em 15 a 20 minutos e os estudantes de medicina têm demonstrado continuado interesseno seu teor, conforme revela o elevado índice de resposta $(98 \%)$ ao longo da duração deste estudo.

Bordage e colaboradores admitiram que o IRD seria útil para identificar potencialidades e limitaçōes dos aprendizes

Tabela 6

Comparação Emparelhada de Rendimento Acadêmico dos Estudantes de Medicina no Quinto e Sexto Períodos do Curso entre Grupos de Classificaçāo do IRD

\begin{tabular}{|c|c|c|c|c|c|}
\hline Grupos de -lessificaşio & $\begin{array}{l}\text { Rendimento } \\
\text { Periodo } 5 \\
\text { Média (dp) }\end{array}$ & $\begin{array}{l}\text { Rendimento } \\
\text { Persodo } 6 \\
\text { Media (dp) }\end{array}$ & $\begin{array}{c}\text { Cotrelação } \\
r\end{array}$ & $\begin{array}{c}\text { Diferença } \\
\text { Média }\end{array}$ & $\begin{array}{c}\text { Testes } t \\
p\end{array}$ \\
\hline$A(n=95)$ & $3,87(0,41)$ & $3,98(0,59)$ & 0,62 & 0,11 & 0,025 \\
\hline$B(n=266)$ & $3,81(0,42)$ & $3,92(0,56)$ & 0,58 & 0,11 & 0,000 \\
\hline$C(n=62)$ & $3,81(0,45)$ & $3,99(0,58)$ & 0,56 & 0,18 & 0,007 \\
\hline$D(n=76)$ & $3,73(0,45)$ & $3,88(0,64)$ & 0,70 & 0,15 & 0,003 \\
\hline$E(n=107)$ & $3,80(0,39)$ & $3,85(0,54)$ & 0,59 & 0.05 & 0,206 \\
\hline$P(n=62)$ & $3,73(0,47)$ & $3,63(0,64)$ & 0,65 & $-0,10$ & 0,102 \\
\hline Total $(\mathrm{N}=668)$ & $3,80(0,43)$ & $3,89(0,60)$ & 0,61 & 0,09 & 0,000 \\
\hline
\end{tabular}

Dados emparelhados ausentes em 9 casos. 
en termos de flexibilidade e estrutura no raciocínio diagnóstico ${ }^{2}$ Os resultados da análise fatorial indicam que os dois componentes aventados são insuficientes para explicar a matriz de intercorrelação entre os itens e a variação de respostas ao IRD. A identificação đe seis fatores de variação no presente estudo revela distinçōes mais extensas entre os aprendizes na composição do processo diagnóstico aferido pelo instrumento, além de ser compatfvel comn o modelo de múltiplas facetas do diagnóstico clínico proposto por Meyer e Cleary ${ }^{17}$.

As diferenças entre os índices do IRD na comparação entre estudantes de duas épocas distintas do curso provavelmente refletem, em parcela maior, efeitos de aceleração da aprendizagem do diagnóstico clínico no evolver do curso. Mudanças de enfoque educativo - tais como a maiox inclusāo de discussão de casos clínicos no ensino de várias disciplinas básicas e o revigoramento da aprendizagem prática e baseada em evidências no ensino de serriologia, clínica médica e clínica cirúrgica - ocorreram em anos mais recentes. $\mathrm{O}$ tamanho do efeito (effect size $=0,4$ ) da época de inserção no indice médio global do IRD foi equivalente àqquele observado na passagem do quinto para o sexto período do curso, conforme dados publicados ${ }^{12}$.

Outro ponto a favor dessa interpretação deriva do fato de que houve diferenças significantes nas dimensões que implicam orientação especffica e experiência clínica (tais como procedimento diligente, apreensão fácil e abstração de significado), mas não naquelas que refletem o perfil psicológico dos estudantes (flexibilidade de concepção e percepção de incerteza). Contudo, efeitos de mudanças na composição do alunado - em termos de grau de autoconfiança, estilo de aprendizagem e distribuição sexual - năo podem ser descartados. Os estudantes da época mais recente revelaram maior grau de autoconfiança como aprendiz, associado principalmente à dimensão de procedimento diligente; já no caso da dimensão de avaliação comparativa, a relação mais estreita foi com a diferença na distribuiçâo sexual.

O efeito diferencial da época de formação se expressa nos grupos de classificação. Os dois grupos ( $\mathrm{A}$ e C), em que houve aumento maior da proporção de estudantes na época mais recente, são aqueles que apresentaram índices mais elevados de procedimento diligente e apreensão fácil, bem como proporçōes mais significativas de nível extenso de autodeterminação percebida no processo diagnóstico. Um estudo anterior mostrou uma associaçāo estreita entre o nivel de autodeterminação percebida e o incremento no escore global do IRD do quinto para o sexto semestre ${ }^{12}$.

Outro aspecto de interesse é a associação entre os grupos de classificaçāo e o gradiente de crescimento no rendimento cognitivo. Os achados sugerem que alguma restrição na maturação do raciocínio diagnóstico (associada em um nivel limitado de eficácia presumida ou de autodeterminação no processo) prevalece entre aprendizes que não evidenciaram ampliação do rendimento cognitivo do quinto para o sexto semestre. Esse fenômeno deriva potencialmente de fatores próprios dos aprendizes (e.g., nivel de reflexão na aprendizagem), ou do contexto de aprendizagem (e.g., compatibilidade com expectativas do estudante) ${ }^{12.18}$.

Em acréscimo ao exposto acima, estudos diversos identificaram uma relação positiva e significante entre os escores do IRD e o nível de experiência clinica ${ }^{2,19}$. Um trabalho anterior revelou que o índice global do IRD era um fator preditivo fraco, mas significante e independente (do nível de conhecimento) do escore de estudantes em teste de resolução de problemas clinicos ${ }^{11}$. Os índices do IRD, embora claramente relacionados ao nivel de experiência clínica, não medem direta* mente a expertise no diagnóstico clínico, segundo dados publicados recentemente. Esses dados mostraram discrepância entre o índice global do IRD e a expertise no diagnóstico, definida em termos de precisão, eficiência e efetividade ${ }^{20}$. Os autores desse estudo indicam que o IRD avalia globalmente $o$ estilo $e$ as atitudes relacionadas ao raciocínio clínico e não necessariamente a expertise no diagnóstico. Os achados do presente estudo permitem especular que a inferência de expertise deve se basear em elementos específicos, antes que no indice global do IRD.

Os dados da atividade de monitoria mostraram um espectro de distribuição peculiar que tem mais correspondência com o quadro de gradiente de rendimento acadêmico do que com o perfil de índices do IRD. Como seria de se esperar, a freqüência de atividade de monitoria tem relação mais estreita com o nfvel de rendimento acadêmico, especialmente $o$ do sexto período do curso no qual os estudantes se envolvem em extensos estudos em clínica médica e clínica cirúrgica. Um dos fatores pertinentes às diferenças encontradas talvez seja o nível de motivação para a aprendizagem, relacionado à preferência por carreira. Nessa fase de iniciaçāo clínica, o nivel de motivação pode ser mais intenso entre os aprendizes que tenham em mente carreiras nas areas de cirurgia e clínica médica do que entre aqueles que visam outras áreas da prática profissional.

Que implicaçôes têm os resultados deste estudo para a instrução acerca do processo de diagnóstico clínico? Os índices globais têm sido preferencialmente utilizados no uso educativo do IRD. Observou-se no presente estudo que o rendimento cognitivo no sexto semestre mostrou correlações mais fortes com as dimensões de apreensão fácil e abstração de sig- 
nificado (que implicam conhecimento elaborado ou construção de esquemas) do que com a dimensāo de procedimento diligente (habitualmente enfatizada na instrução clínica). Esse achado ê realçado por evidências acumuladas nos últimos anos que sugerem a predominância variável de mês modos de processo cognitivo no diagnóstico clínico: raciocínio hipotéticodedutivo, raciocínio esquema-indutivo e reconhecimento de arquétipos (pattern recognition) 2 $^{21}$. Aprendizes e peritos possivelmente adotam uma combinação variável de modos dependendo do tipo de problema clínico, do nível de conhecimento pertinente ao problema, da etapa na busca de resoluçăo e do contexto de desempenho em que se encontram. Coderre e colaboradores sugeriram recentemente que as estratégias educacionais deveriam favorecer a aprendizagem do modo esquema-indutivo de raciocínio diagnóstico na iniciação clínica ${ }^{22}$

Os resultados descritos neste trabalho sāo consistentes com a noçāo de que a perspectiva do processo diagnóstico, embutida no IRD, envolve distintos elementos e suas medidas refletem, em grau variável, o conhecimento elaborado e a experiência clínica, a percepção da eficácia pessoal e o perfil de conduta individual (em termos de interação interpessoal, motivação e processo decisório) no encontro clínico. Os achados implicam que os estudantes diferem no entendimento e no modo do processo diagnóstico, ainda que demonstrem receptividade à orientação de aprendizagem desse processo.

Em conclusão, este estudo examinou as relaçōes e distribuição dos índices das dimensões do Inventário de Raciocinio Diagnóstico na populaçāo-alvo de estudantes de medicina envolvidos na situaçāo real de aprendizagem clínica. Verificou-se que os estudantes diferem nas percepçס̄es do processo diagnóstico, inclusas no IRD, e as percepções configuradas têm relaçōes significativas com o progresso na aprendizagem e as opçôes de aprofundamento e aplicação do aprendizado.

\section{AGRADECIMENTOS}

O autor agradece a participação ativa e 0 interesse real dos aprendizes envolvidos.

\section{REFERENCIAS BIBLIOGRAFICAS}

1. Grant J, Marsden P. The structure of memorised knowledge in students and clinicians: an explanation for diagnostic expertise. Med Educ 1987; 21: 92-98.

2. Bordage G, Grant J, Marsden P. Quantitative assessment of diagnostic ability. Med Educ 1990; 24: 413-425.

3. Bordage $G$, Zacks $R$. The structure of medical knowledge in the memory of medical students and general prac- titioners: categories and prototypes. Med Educ 1984; 18: 406-411.

4. Bordage G, Lemieux M. Semantic structures and diagnostic thinking of experts ad novices. Acad Med 1991; 66: S70S72.

5 Gale J, Marsden P. Medical diagnosis: from student to clinician. Oxford: Oxford University Press, 1983.

6. Grant J, Marsden P. Primary knowledge, medical education and consultant expertise. Med Educ 1988; 22: 173-179.

7. Sobral DT. Diagnostic ability of medical students in relation to their characteristics and preclinical background. Med Educ 1995; 29: 278-282.

8. Jones UF. The reliability and validity of the Bordage, Grant \& Marsden inventory for use with physiotherapists. Med Teacher 1997; 19: 133-140.

9. Peterson C. Factors associated with success or failure in radiological interpretation: diagnostic-thinking approaches. Med Educ 1999; 33: 251-259.

10. Round AP. Teaching clinical reasoning - a preliminary controlled study, Med Educ 1999; 33; 480-483.

11. Sobral DT. Características do Inventário de Raciocínio Diagnóstico de Bordage, Grante Marsden. Psicol: Teoria Pesq 2001; 17: 79-85.

12. Sobral DT. Avaliação da maturação do processo de raciocínio diagnóstico de estudantes de medicina. Medicina, Ribeirão Preto 2001; 34: 89-99.

13. Bandura A. The nature and structure of self-efficacy. In; Bandura A. Self-efficacy. The exercise of control. New York: WH Freeman, 1997, p. 36-78.

14. Hulley SB, Cummings SR. Designing clinical research. Baltimore: Williams and Wilkins, 1988.

15. Norman GR, Streiner DI. Biostatistics: the bare essentials. St. Louis: Mosby, 1994.

16. Glantz AS, Slinker BK. Primer of applied regression analysis and variance. New York: McGraw-Hill, 1990.

17. Meyer\}HF, Cleary EG. An exploratory student learning model of clinical diagnosis. Med Educ 1998; 32: 574-581.

18. Sobral D.T. An appraisal of medical students' reflectionin-learning. Med Educ 2000; 34: 182-187.

19. Groves, M, Scott I \& Alexander $H$. Assessing clinical reasoning: a method to monitor its development in a PBL curriculum, Med Teacher 2002; 24: 507-515.

20. Groves $\mathrm{M}, \mathrm{O}^{\prime}$ Rourke $\mathrm{P}$, Alexander $\mathrm{H}$. The clinical reasoning characteristics of diagnostic experts. Med Teacher 2003; 25: 308-313. 
筑: Norman GR, Eva KW. Doggie diagnosis, diagnostic success and diagnostic reasoning strategies: an alternative view. Med Educ 2003; 37: 676-677.

22. Coderre S, Mandin H, Harasym PH, Fick GH. Diagnostic reasoning strategies and diagnostic success. Med Educ 2003; 37: 695-703.

\section{Correspondência}

D.T. Sobral

Universidade de Brasilia

C.P. 04569 Medicina

70919-970

E-mail: dtsobral@unb.br 